\title{
Probabilidad de incumplimiento en inversiones de infraestructura: análisis a partir de modelos estructurales de riesgo de crédito
}

\author{
ZAPATA QUIMBAYO, CARLOS ANDRÉS \\ Observatorio de Economía y Operaciones Numéricas - ODEON \\ Universidad Externado de Colombia \\ Correo electrónico: carlosa.zapata@uexternado.edu.co
}

\begin{abstract}
RESUMEN
Este trabajo tiene como objetivo estimar las probabilidades de incumplimiento en proyectos de infraestructura. Para ello, se analiza la exposición que tienen los prestamistas frente a un estado de incumplimiento. Esta aplicación se realiza al asumir dinámica propia del ratio de cobertura del servicio de la deuda (DSCR) y el perfil de pago determinado por el flujo de efectivo disponible del proyecto, donde estos se modelan estocásticamente siguiendo los mismos supuestos de la teoría de valoración de opciones desarrollado por Black y Scholes (1973) y Merton (1973). De esta forma, mediante la adaptación de modelos estructurales desarrollados para activos ilíquidos, como extensión de los modelos de riesgo de crédito de Merton (1974) y KMV de Moody's, se analizan los componentes de probabilidad, exposición y pérdidas de los prestamistas en escenarios de incumplimiento.
\end{abstract}

Palabras clave: riesgo de crédito, probabilidad de incumplimiento, proceso estocásticos. Clasificación JEL: C14; C63; G13.

MSC2010: 60H30; 68U20. 


\title{
Probability of default in infrastructure projects: analysis from structural models of credit risk
}

\begin{abstract}
The purpose of this paper is to estimate the default probabilities in infrastructure projects. For that, we analyze the exposure of the lenders to a state of default. This application is made by assuming the debt service coverage ratio (DSCR) dynamic itself and the payment profile determined by the available cash flow of the project, where these are stochastically modeled following the same assumptions of the valuation theory of options developed by Black and Scholes (1973) and Merton (1973). In this way, through the adaptation of structural models developed for illiquid assets, as an extension of the credit risk models of Merton (1974) and KMV of Moody's, the probability, exposure and loss components of the lenders are analyzed in scenarios of default.
\end{abstract}

Keywords: credit risk, default probability, stochastic processes.

JEL classification: C14; C63; G13.

MSC2010: 60H30; 68U20.

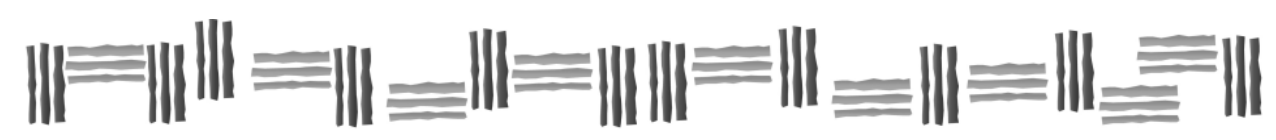




\section{Introducción.}

La financiación de proyectos o Project Finance (PF) corresponde a una técnica de financiación estructurada utilizada ampliamente en países desarrollados y en desarrollo a través de esquemas de asociación público-privada (APP) e iniciativas de financiación privada (IFP). Las limitaciones de los recursos y/o restricciones en la capacidad de endeudamiento, junto con la necesidad de mejorar y ampliar la provisión de bienes y servicios públicos (Boussabaine, 2013), así como la transferencia de riesgos y su adecuada gestión (Grimsey y Lewis, 2002; Gatti, 2008; Gatti et al., 2007), ha generado una necesidad creciente para que los gobiernos acudan ante inversionistas privados para participar en estos esquemas de financiación a largo plazo. Para ello, el gobierno (o la autoridad pública) concede a un grupo de inversionistas privados (consorcios o concesiones) el diseño, construcción, operación y mantenimiento de grandes proyectos de infraestructura dentro de un acuerdo contractual de largo plazo (Gatti, 2008; Buscaino et al., 2012).

De esta forma, la financiación de proyectos se ha venido consolidado como una práctica de mayor intensidad y representa una herramienta útil para ayudar a reducir las brechas que presentan los países en materia de infraestructura. Según Della y Yermo (2013), el sector de infraestructura ha representado más del $60 \%$ de los préstamos globales de financiación de proyectos. Bajo este esquema se crea un vehículo de propósito especial (SPV, por su sigla en inglés) sobre una base ad hoc (Yescombe, 2002) y representa una compleja estructura de financiación con carácter independiente respecto a los inversionistas y con recursos limitados de estos (Gatti, 2008), los cuales determinan un esquema de financiación fuera de balance con un alto nivel de apalancamiento y largos periodos para recuperar la inversión. Este tipo de financiación también puede incorporar derechos de control (significativos y extensos) para los prestamistas (Borgonovo y Gatti, 2013; Blanc-Brude, Hasan e Ismail, 2014), así como covenants y clausulas restrictivas (Gatti, 2008; Blanc-Brude, Hasan y Whittaker, 2016), los cuales limitan los intereses de los inversionistas.

A diferencia de la financiación corporativa tradicional, todas las consecuencias económicas del proyecto son atribuidas directamente al SPV (Gatti, 2008). Por tal motivo, la dirección del proyecto debe orientar gran parte de sus esfuerzos a la generación del flujo de efectivo, buscando así cubrir las obligaciones contraídas, es decir el reembolso de la deuda y para remunerar el capital invertido, una vez el proyecto ha cubierto sus requerimientos operativos (costos, mantenimiento, gastos, impuestos, entre otros).

Además, la capacidad que tiene el proyecto para generar flujo de efectivo determina su capacidad crediticia. Sin embargo, el análisis crediticio en los proyectos de infraestructura termina siendo mucho más complejo, en comparación con el sector corporativo. El problema radica en la incertidumbre futura sobre el éxito del proyecto, dado los diferentes riesgos que están presentes en sus fases del ciclo de vida, por ejemplo, una demora en la terminación de la construcción por eventos naturales o sociales, una reducción de su demanda o de sus ingresos, un aumento de los costos operativos, entre otros (Cartea y Figueroa, 2005; Boussabaine, 2013). Además, cualquier incumplimiento de las obligaciones contractuales de las contrapartes puede interrumpir el correcto funcionamiento del proyecto. Esta incertidumbre se traduce en la probabilidad de incumplir los pagos acordados en el contrato de financiación (Borgonovo y Gatti, 2013). A esto se suma la ausencia de antecedentes crediticios del proyecto que sirvan como base para una decisión de financiación (Yescombe, 2002).

Blanc-Brude y Strange (2007) señalan que este tipo de financiamientos presentan una mezcla de diferentes tipos de deuda como deuda senior, mezzanine y subordinada, además de préstamos puente e instalaciones de reserva, cada una con diferentes vencimientos y ordenes de prelación de pago. Es necesario aclarar que el análisis correspondiente debe realizarse para cada uno de los diferentes niveles de deuda: senior, mezzanine y subordinada; dada las características de estos y la prelación de pago. Al respecto, Freydefont (2001) encuentra que el riesgo de crédito en un componente de deuda, por ejemplo, deuda senior, es particular e independiente de los demás componentes (mezzanine y/o subordinada) de un orden, pero estos últimos están determinados por la deuda con prelación de pago. Aunque su 
representación puede ser compleja, la construcción de un modelo financiero que defina la cascada de pagos correspondiente puede llevar a una implementación intuitiva y práctica, como indica Gatti (2008).

Un análisis rigoroso del riesgo de crédito requiere, por tanto, reflejar estas características. A pesar de la importancia práctica que representa para la banca, el análisis y evaluación crediticia de los proyectos ha recibido poca atención en la literatura financiera. Además, los modelos tradicionales de evaluación crediticia: i) modelos de forma reducida; ii) modelos basados en calificaciones y, iii) modelos estructurales, no pueden aplicarse directamente o presentan serias limitaciones para este tipo de financiaciones (Klompjan \& Wouters, 2002; Gatti et al., 2007; Kong et al., 2008; Dong et al., 2012; Karminsky \& Morgunov, 2016). A pesar de la ventaja que ofrecen los modelos estructurales, donde se modela el mecanismo por el que ocurren los incumplimientos en contraste con la ocurrencia de eventos predeterminados como ocurre en los primeros dos enfoques, estos no pueden aplicarse directamente y, por tanto, deben ajustarse para incorporar las características distintivas de los proyectos de infraestructura.

Klompjan y Wouters (2002) y Karminsky y Morgunov (2016) encontraron limitaciones asociadas a la poca disponibilidad de datos sobre incumplimientos (históricos) y/o a las dificultades de acceso a esta información, así como a la diversidad en el propósito de cada proyecto y de sus riesgos, lo que dificulta la determinación de las variables explicativas dentro del modelo. Klompjan y Wouters (2002), a partir de información interna de ING sobre financiaciones de proyectos realizadas entre 1995 y 1997, desarrollan un modelo Logit para identificar los factores que están fuertemente asociados con el riesgo de incumplimiento de un proyecto. Los autores encontraron que el uso de tecnología no probada, la presencia de patrocinadores inexpertos, los bajos niveles de cobertura del servicio de la deuda están asociados a un mayor riesgo de incumplimiento. De forma similar, Karminsky y Morgunov (2016) utilizaron la información interna de ING durante el periodo 2007-2013 para evaluar aquellos factores que determinan la probabilidad de incumplimiento.

Kong et al. (2008) proponen un modelo de estimación la probabilidad de incumplimiento y de la pérdida crediticia a partir del uso de matrices de transición condicionales de calificación crediticia para proyectos de autopista. Para cuantificar el riesgo de crédito, los autores asumieron que solo hay cuatro factores de crédito y que la tasa de recuperación era nula en caso de incumplimiento. Además, ellos asumieron que los prestamistas solo inviertan en un solo proyecto a la vez, lo que es inconsistente con la práctica. Estas limitaciones son señaladas por Dong et al. (2012) quienes argumentan que los prestamistas están dispuestos a participar simultáneamente en diferentes proyectos con el objetivo de diversificar los riesgos idiosincrásicos de cada uno. Dong et al. (2012) proponen un modelo basado en copulas para portafolios de proyectos correlacionados junto con la técnica de simulación de Monte Carlo. Su aplicación busca extender el modelo desarrollado por Gatti et al. (2007) utilizado para estimación del valor en riesgo $(\mathrm{VaR})$ de un acuerdo de financiación de proyectos.

Desde el enfoque de modelos estructurales, Freydefornt (2001) desarrolla un modelo de estimación del riesgo de crédito para proyectos de infraestructura, como una extensión del modelo de Merton (1974), al asumir que los pasivos del proyecto pueden considerarse como reclamaciones contingentes sobre los activos. De esta forma, estima la pérdida esperada y las primas de riesgo para todas las categorías de deuda. Por su parte, Aragones, Blanco e Iniesta (2009) adoptan el modelo KMV de Moody's, para estimar el riesgo de crédito en proyectos de infraestructura. Sin embargo, los autores realizan una estimación directa de este buscando diferenciarlo del enfoque denominado por ellos como project finance, que se centra en determinar si la generación de los flujos de efectivo del proyecto es capaz de asegurar el pago de la deuda.

Aunque los modelos estructurales de Merton (1974) y KMV de Moody's, como muestran Freydefornt (2001) y Aragones, Blanco e Iniesta (2009), tienen una ventaja frente a los enfoques anteriores aún persisten limitaciones fuertes en su implementación como señalan Blanc-Brude y Hasan (2016). Para superar las dificultades anteriores, Blanc-Brude y Hasan (2016) proponen para estimar la probabilidad de incumplimiento en proyectos de infraestructura a partir de un modelo estructural desarrollado para deuda ilíquida, como una extensión de modelo KMV de Moody's al redefinir la 
distancia al incumplimiento. A diferencia de las dos aplicaciones anteriores, ellos estiman la probabilidad de incumplimiento a partir de un tratamiento estocástico en la dinámica del perfil pago del proyecto, que se determina a través del flujo de efectivo disponible. Además, su modelo incorpora no solo los efectos de los covenants de deuda sino también la naturaleza dinámica de la capacidad de pago del proyecto al asumir una dinámica estocástica de las ratios de cobertura del servicio de la deuda.

La principal innovación de este enfoque se debe a la incorporación de una dinámica es propia de la ratio de cobertura del servicio de deuda ( $D S C R$, por su sigla en inglés), donde éste se modela estocásticamente siguiendo los mismos supuestos de la teoría de valoración de opciones desarrollado por Black y Scholes (1973) y Merton (1973). Este modelo fue extendido por Blanc-Brude et al. (2018) para estimar la probabilidad condicional de transición entre estados (de un estado de riesgo a uno seguro $\mathrm{y}$, viceversa) al integrar técnicas de inferencia bayesiana.

Partiendo de los modelos estructurales de riesgo de crédito, este trabajo tiene como objetivo estimar las probabilidades de incumplimiento en proyectos de infraestructura y se analiza la exposición y pérdidas que tendrían los prestamistas frente a un incumplimiento. Para ello, al igual que Blanc-Brude y Hasan (2016) y Blanc-Brude et al. (2018), se asume una dinámica estocástica del perfil de pago del proyecto determinado por el flujo de efectivo disponible. De esta forma, se adopta un modelo estructural de riesgo de crédito desarrollado para deuda ilíquida. Esta metodología representa una extensión de los modelos tradicionales de riesgo de crédito de Merton (1974) y KMV de Moody's.

A parte de esta introducción, este trabajo está organizado de la siguiente forma. En la segunda sección se ofrece una breve descripción del riesgo de crédito en proyectos de infraestructura, su definición y naturaleza. En la tercera sección se presentan los modelos estructurales de estimación del riesgo de crédito y su adaptación al campo de los proyectos de infraestructura basado en la propuesta de Blanc-Brude y Hasan (2016). Luego, se presenta una aplicación sencilla y se analizan los resultados. Finalmente, se presenta una discusión final y conclusiones.

\section{Riesgo de crédito y modelos estructurales de estimación.}

La definición de la probabilidad de incumplimiento, así como la cuantificación del riesgo de crédito en el sector corporativo, han sido ampliamente estudiadas en la teoría financiera, donde la evaluación crediticia se realiza a partir de la información financiera que proveen las hojas de balance y los estados financieros, así como su desempeño histórico y el éxito de financiaciones previas (Gatti et al., 2007). De esta forma, la información disponible es suficiente para estimar la pérdida esperada por riesgo de crédito (EL, por su sigla en inglés), a partir de los tres componentes definidos por el Comité de Basilea en el acuerdo de Basilea II, como indica la ecuación (1):

$$
E L=D P \times L G D \times E A D
$$

donde, $D P$ es la probabilidad de incumplimiento, $L G D$ es la pérdida dado el incumplimiento y $E A D$ la exposición al incumplimiento. Cabe resaltar que gran parte de los desarrollos teóricos se han enfocado en la estimación del primer componente, es decir, la $D P$, en este campo los modelos estructurales han ofrecido una ventaja considerable (ver Freydefornt, 2001; Aragones, Blanco \& Iniesta, 2009; Blanck-Brude \& Hasan, 2016; Blanc-Brude, Hasan \& Whittaker, 2018). Sin embargo, los demás componentes de estimación de la $E L$, pueden tener limitaciones que son importantes y merecen una atención especial. Por lo general, los prestamistas, en su esfuerzo de reducir su exposición al riesgo, exigen al $S P V$ la creación de cuentas de reserva de efectivo para cubrir parte del servicio de deuda futura (Gatti, 2008), buscando aumentar su tasa de recuperación y reducir la exposición total en caso de un evento de incumplimiento.

A partir de los trabajos seminales de Black y Scholes (1973) y Merton (1973), los modelos de valoración de opciones financieras han sido adaptados para el tratamiento de problemas corporativos, 
entre ellos la evaluación del riesgo de crédito. Este campo comprende un enfoque de estimación a partir de modelos estructurales iniciado por Merton (1974) y, sus posteriores desarrollados presentados por Black y Cox (1976) e Ingersoll (1977), este enfoque se conoce como "el modelo de Merton". Este modelo ha sido ampliamente utilizado para estimar la probabilidad de incumplimiento $(D P)$.

Merton (1974) extendió la fórmula de Black-Scholes al análisis de reclamaciones contingentes ( $C C A$, por su sigla en inglés) para el tratamiento de problemas corporativos, donde asume que la deuda de una empresa puede ser considerada como una reclamación sobre los activos de la misma, con un precio de ejercicio igual a su valor nominal y una fecha de vencimiento determinada. De esta forma, propone una relación entre la estructura de capital y la capacidad que tiene la empresa para pagar su deuda. Para ello, asume que la evolución del valor de mercado de los activos de la empresa $\left(V_{A}\right)$ sigue un proceso estocástico del tipo movimiento geométrico browniano $(M G B)$ como indica la ecuación (2):

$$
d V_{A}=\mu_{A} V_{A} d t+\sigma_{A} V_{A} d W_{t}
$$

donde, $\mu_{A}$ reprsenta a la tasa de deriva del valor de los activos de la empresa, $\sigma_{A}$ su volatilidad, y $W_{t \in[0, T]}$ es un proceso estándar de Wiener definido sobre un espacio de probabilidad $(\Omega, \mathcal{F}, P)$ con una filtración $\left(\mathcal{F}_{t}\right)_{t \in[0, T]}$. Ahora, si el valor de los activos de la empresa $\left(V_{A}\right)$ sigue un proceso lognormal como indica la ecuación (2), se tiene entonces:

$$
\ln V_{A}=\ln V_{0}+\left(\mu-\frac{1}{2} \sigma_{A}^{2}\right) t+\sigma_{A} Z \sqrt{T}
$$

donde, $V_{0}$ representa el valor actual de los activos de la empresa y $Z \sim N(0,1)$. Además, el valor de sus activos, está determinado por $V_{A}=V_{E}+V_{D}$ en un momento inicial $t=0$, donde $V_{E}$ es el valor de mercado de las acciones de la empresa y $V_{D}$ es el valor de su deuda.

El modelo asume que $V_{D}$ está representada por la emisión de un bono cero cupón, con vencimiento en $T$ y valor $B(t, T)$. Entonces, si $V_{T}<V_{D}$ la empresa incumple con el pago de su deuda, en este caso $V_{E}=0$, mientras que, si $V_{T} \geq V_{D}$, la empresa paga su deuda en $T$ y, por tanto $V_{E}=V_{T}$ $V_{D}$. Esta lógica se puede representar como una función de pagos dada por

$$
V_{E}=\max \left(V_{T}-V_{D} ; 0\right)
$$

De esta forma, $V_{E}$ se asimila a una opción de compra de tipo europea con precio de ejercicio igual $V_{D}$. Lo anterior indica que si el valor de los activos es insuficiente para cumplir con los pasivos, entonces los accionistas, titulares de la opción de compra, no ejercerán su derecho y dejarán la empresa a sus acreedores.

Por su parte, la probabilidad de incumplimiento de la empresa $(\operatorname{Prob}(0, T))$ definida entre $t=0$ y $T$, está determinada por:

$$
\operatorname{Prob}(0, T)=\operatorname{Pr}\left[V_{A} \leq V_{D}\right]=\operatorname{Pr}\left[\ln V_{A} \leq \ln V_{D}\right]
$$
Scholes:

Entonces, en un mundo riesgo-neutral el valor de $V_{E}$ está determinado por la fórmula de Black-

$$
V_{E}=V_{A} N\left(d_{1}\right)-V_{D} e^{-r T} N\left(d_{2}\right)
$$

donde $r$ representa la tasa de interés libre de riesgo y $N(\cdot)$ indica la función de distribución normal acumulada de los parámetros $d_{1}$ y $d_{2}$, que vienen dados por:

$$
d_{1}=\frac{\ln \left(\frac{V_{A}}{V_{D}}\right)+\left(r+\frac{1}{2} \sigma_{A}^{2}\right) T}{\sigma_{A} \sqrt{T}}
$$




$$
d_{2}=\frac{\ln \left(\frac{V_{A}}{V_{D}}\right)+\left(r-\frac{1}{2} \sigma_{A}^{2}\right) T}{\sigma_{A} \sqrt{T}}=d_{1}-\sigma_{A} \sqrt{T}
$$

Finalmente, la probabilidad de incumplimiento (en un mundo riesgo-neutral) es la probabilidad de que $V_{A}$ en $T$ se encuentre por debajo de $V_{D}$ :

$$
P\left[V_{A} \leq V_{D}\right]=N\left(-d_{2}\right)
$$

Una limitación importante del modelo de Merton (1974) es que los parámetros del proceso determinado por la ecuación (2), es decir, $\mu_{A} \mathrm{y} \sigma_{A}$, no son directamente observables, lo que dificulta su aplicación. Como propuesta para superar esta limitación, Vasicek (1984) adoptó un enfoque novedoso para la implementación del modelo de Merton, conocido actualmente como modelo KMV de Moody's, que ha demostrado tener un éxito considerable en la medición del riesgo de crédito (Kealhofer, 1993).

El modelo KMV, como extensión del modelo Merton, busca estimar la probabilidad de incumplimiento de una empresa basado en la noción de distancia al incumplimiento $(D D$, por su sigla en inglés), al asumir que la empresa está en situación de incumplimiento cuando el valor de sus activos está por debajo de un umbral definido por el valor de su deuda (Figura 1). Esta diferencia se fundamenta en la definición del punto de incumplimiento ( $D P$, por su sigla en inglés).

La Figura 1 muestra que si el valor de los activos cae por debajo del punto de incumplimiento, entonces la empresa incumple el pago de su deuda. Por lo tanto, la probabilidad de incumplimiento es la probabilidad de que el valor del activo se encuentre por debajo de este punto. Esta probabilidad esta representada en el área sombreada de la función de distribución debajo del punto de incumplimiento.

Figura 1. Estimación de la probabilidad incumplimiento.

\author{
Valor de \\ de los activos
}

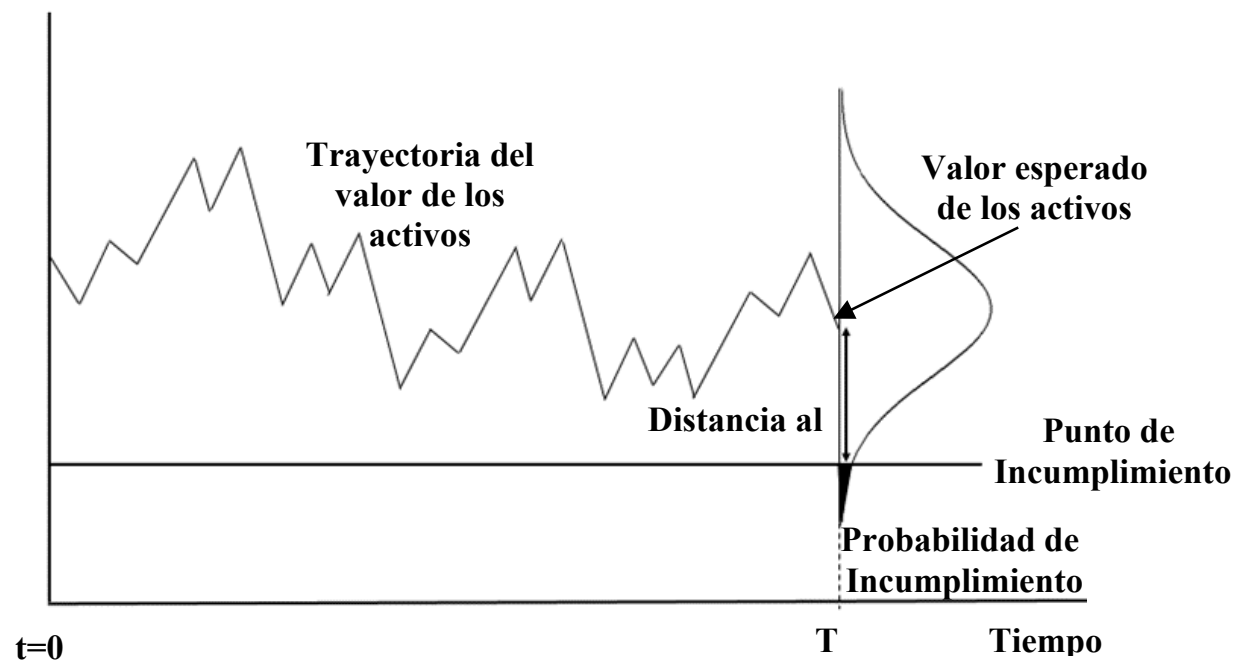

Fuente: Elaboración propia, basado en Kealhofer (2003).

Como resultado, el modelo estima la distancia al incumplimiento definido como el número de desviaciones estándar en que el valor de los activos supera el punto de incumplimiento. Entonces, la distancia al incumplimiento $(D D)$ se calcula como:

$$
D D=\frac{V_{A}-D P}{\sigma_{A} V_{A}}
$$


donde, $V_{A}$ representa el valor de los activos y $\sigma_{A}$ su volatilidad. Para una empresa con responsabilidad limitada, su patrimonio el cual está determinado por el valor de mercado de las acciones $\left(V_{E}\right)$, tiene el derecho residual sobre los activos después de que se hayan cumplido todas las demás obligaciones. De esta forma, una opción de compra sobre los activos con precio de ejercicio igual a la deuda, tiene las mismas propiedades indicadas en el modelo de Merton. Al tomar los mismos supuestos sobre $V_{A}, V_{E}$ y $V_{D}$, dados por las ecuacones (2) y (6), $D D$ se podría estimar usando la ecuación (7b) como:

$$
D D=\frac{\ln \left(\frac{V_{A}}{V_{D}}\right)+\left(r-\frac{1}{2} \sigma_{A}^{2}\right) T}{\sigma_{A} \sqrt{T}}
$$

donde el numerador de la ecuación (10) determina el nivel de apalancamiento financiero, que está asociado al nivel de riesgo financiero que tiene la empresa, mientras que el denominador refleja su nivel de riesgo de mercado. Sin embargo, como $\sigma_{A}$ no es observable, $D D$ se aproxima mediante la ecuación (9). Mientras que la probabilidad de incumplimiento (riesgo-neutral) en un vencimiento $T$, está determinada por la ecuación (11):

$$
P\left[V_{A} \leq V_{D}\right]=N(-D D)
$$

Como resultado, este modelo establece que $D D$ es suficiente para estimar la probabilidad de incumplimiento. Sin embargo, se debe tener presente que, en proyectos de infraestructura, el tratamiento del riesgo de crédito es mucho más complejo. Estas diferencias fueron señaladas por el Comité de Basilea desde su primera versión (Gatti, 2008). A partir de la distinción entre la financiación corporativa y la financiación estructurada de proyectos, el Comité de Basilea recomendó la implementación de metodologías diferentes para determinar la $E L$ tanto para el modelo estándar como para los modelos internos (IRB, por su sigla en inglés). Desde entonces se han hecho pocos esfuerzos para definir una metodología estándar que permita hacer frente a este problema; sin embargo, en gran parte de éstas no se han marcado las diferencias entre los tipos de financiación; i) financiación tradicional o corporativa y ii) financiación estructurada de proyectos.

A pesar de los desarrollos recientes de la teoría financiera en la evaluación del riesgo de crédito para el sector corporativo, el desarrollo y aplicación de modelos para proyectos de infraestructuras ha sido muy escaso. Cabe tener presente que, en estos proyectos, el flujo de efectivo del proyecto es el factor determinante de su valor y la determinación de su capacidad de pago, por tanto, éste es el único referente para su aplicación (Gatti, 2008). En este ámbito, la propuesta de Blanc-Brude y Hasan (2016) en la que proponen estimar la probabilidad de incumplimiento a través de un modelo estructural desarrollado para deuda ilíquida, como una extensión de modelo KMV de Moody's, representa una propuesta innovadora al redefinir los parámetros del modelo teniendo en cuenta las características distintas de los proyectos de infraestructura.

De esta forma, el riesgo de crédito está asociado a la incertidumbre futura de los flujos de efectivo del proyecto, por lo que su dinámica determina la capacidad de pago y la probabilidad de entrar en una situación de incumplimiento. Por tanto, la evaluación del riesgo de crédito en proyectos está determinado por la posibilidad de que, en un momento dado, los flujos de efectivo sean suficientes o no para pagar el servicio de la deuda.

\section{Estimación de la probabilidad de incumplimiento en proyectos de infraestructura.}

Blanc-Brude et al. (2014) y Blanc-Brude y Hasan (2016) demostraron que los modelos estructurales pueden utilizarse para estimar el riesgo de crédito en proyectos de infraestructura; sin embargo, estos modelos merecen un tratamiento especial y algunos ajustes. Por ejemplo, la incorporación de derechos de control para los prestamistas, así como covenants de deuda, incorporan un efecto importante en la 
estimación de los componentes de estimación del riesgo de crédito. De igual forma, es necesaria una redefinición del punto de incumplimiento.

Mientras los modelos de Merton y KMV definen el incumplimiento a partir del valor de los activos y de la deuda en un horizonte de tiempo determinado $(T)$, para un proyecto el incumplimiento debe estimarse para cada periodo $(t)$. Lo anterior indica que el modelo debe definirse en términos del flujo de efectivo y éste a su vez determina la capacidad de pago al compararse directamente con el servicio de la deuda en cada instante. Esto implica trabajar directamente con la ratio de cobertura del servicio de deuda ( $D S C R$, por su sigla en inglés). A partir de este ajuste, Blanc-Brude y Hasan (2016) demostraron que comprender la dinámica del $D S C R$, junto con el perfil de amortización de la deuda y las garantías disponibles son suficientes para implementar un modelo estructural de riesgo de crédito. Según los autores, la presencia de estas características distintivas hace que los modelos estructurales de riesgo de crédito sean una opción natural. Su implementación puede hacerse mediante tres pasos:

1. modelación de los flujos de efectivo y del $D S C R$ para cada momento $t$,

2. ajuste riesgo-neutral y estimación de la probabilidad de incumplimiento,

3. incorporación de escenarios de reestructuración y/o refinanciación de la deuda.

El primer paso para estimar el riesgo de crédito corresponde a la modelación de los flujos de efectivo para cada momento $t$, donde el incumplimiento se da en aquellos escenarios cuando este es insuficiente para pagar el servicio de deuda (capital e intereses). Así, la determinación de la capacidad de pago del proyecto a partir del DSCR es fundamental para su aplicación. El DSCR mide la cantidad de efectivo disponible para realizar el pago del servicio de la deuda ( $C F A D S$, por su sigla en inglés) en un período $t$, y se estima como:

$$
D S C R_{t}=C F A D S_{t} / D S_{t}
$$

donde $D S_{t}$ representa el servicio de deuda en cada $t$. Cuanto más alto sea el $D S C R$, más efectivo disponible tendrá el proyecto para cumplir con sus obligaciones de deuda. La dinámica de DSCR permite la implementación del modelo estructural de riesgo de crédito, ya que los niveles de DSCR determinan los umbrales de incumplimiento por definición. De esta forma se puede dar una definición inequívoca del punto de incumplimiento (estricto), determinado en $D S C R=1$; o una definición del punto de incumplimiento (técnico), determinado en $D S C R=1, x$. Por tanto, un proyecto puede considerarse en incumplimiento 'estricto' si su $D S C R$ cae por debajo de 1. De esta forma, el conocimiento de la dinámica de $D S C R$ es suficiente para estimar la distancia al incumplimiento $(D D)$.

Dado que el perfil del $D S C R$ está fuertemente relacionado con el riesgo (total) del proyecto, el conocimiento de la distribución del DSCR, junto con el servicio de deuda y la volatilidad de los flujos de efectivo son suficientes para la cuantificación del riesgo de crédito. Si se asume un proceso lognormal que caracteriza la dinámica estocástica del $D S C R$ en cada $t$, se tiene:

$$
d D S C R_{t} /_{D S C R_{t}}=\mu d t+\sigma d W_{t}
$$

donde $\mu$ y $\sigma$ representan la tasa de deriva del $D S C R$ y su correspondiente volatilidad, y se asume que estas son conocidas, por su parte, $W_{t \in[0, T]}$ es un proceso estándar de Wiener. Ahora, como el punto de incumplimiento (estricto) está determinado por:

$$
\operatorname{DSCR}_{t} \equiv C F A D S_{t} /_{D S_{t}}<1,0
$$

Por su parte, la implementación a partir de la definición de incumplimiento técnico solo requiere ajustar la ratio de cobertura en $\mathrm{DSCR}=1, \mathrm{x}$. 
Entonces, de forma análoga al modelo KMV, la distancia al incumplimiento en cada $t$ es:

$$
D D_{t}=\frac{C F A D S_{t}-D S_{t}}{\sigma_{C F A D S} C F A D S_{t}}
$$

donde $\sigma_{C F A D S}$ es la volatilidad de los $C F A D S$. Este parámetro comprende un insumo importante para la implementación del modelo. Sin embargo, su estimación puede realizarse utilizando las mismas técnicas imprentadas en la teoría de opciones reales.

Ahora, con el propósito de evitar cualquier problema debido a la dependencia de escala del CFADS, el flujo de efectivo se reexpresa en términos de DSCR:

$$
C F A D S_{t}=D S C R_{t} \times D S_{t}
$$

Al usar esta definición del CFADS, la distancia al incumplimiento se puede expresar como:

$$
D D_{t}=\frac{1}{\sigma_{C F A D S}}\left(1-\frac{1}{D S C R_{t}}\right)
$$

De igual forma, la ecuación (17) se puede reescribir como una función exclusiva del DSCR expresando la volatilidad de los $C F A D S$ en función de la volatilidad del $D S C R\left(\sigma_{D S C R}\right)$ :

$$
D D_{t}=\frac{1}{\sigma_{D S C R}} \frac{D S_{t-1}}{D S_{t}}\left(1-\frac{1}{D S C R_{t}}\right)
$$

donde $\sigma_{C F A D S}=\left(\frac{D S_{t-1}}{D S_{t}}\right) \sigma_{D S C R}$. Cabe resaltar que sí, dadas las condiciones del crédito, se adopta un esquema de amortización con cuota fija, entonces: $\sigma_{C F A D S}=\sigma_{D S C R}$, dado que $D S_{t-1}=D S_{t}$, para todo el periodo.

De igual forma, la probabilidad de incumplimiento $[P(t, T)]$ viene determinada por $D D_{t}$ :

$$
P(t, T)=N\left(-D D_{t}\right)
$$

Sin embargo, esta medida incorpora las preferencias de riesgo de los inversionistas. Dado que la ecuación (13) muestra la dinámica del $D S C R$ en términos de la tasa de deriva $\left(\mu_{t}\right)$, la definición de la probabilidad de incumplimiento requiere incorporar un ajuste riesgo-neutral determinado por la prima por riesgo $(\lambda)$.Este ajuste riesgo-neutral tiene como objetivo incorporar las preferencias de riesgo de los inversionistas en la modelación del $C F A D S$, pero el desarrollo de una valoración usando la tasa libre de riesgo. Lo anterior indica que si los flujos de efectivo reflejan la naturaleza de riesgo de la inversión, éstos deben ser descontados a la tasa libre de riesgo en el modelo de valoración. De esta forma se tiene:

$$
Q(t, T)=N\left(N^{-1}[P(t, T)+\lambda)\right]
$$

donde $Q(t, T)$ ahora es la probabilidad de incumplimiento riesgo-neutral y $\lambda$ es la prima por riesgo, conocida como coeficiente de Sharpe para un horizonte $T: \lambda=\frac{\mu-r}{\sigma} \sqrt{T}$. Este ajuste riesgoneutral tiene como objetivo incorporar las preferencias de riesgo de los inversionistas en la modelación del CFADS, pero el desarrollo de una valoración usando la tasa libre de riesgo. Lo anterior indica que, si los flujos de efectivo reflejan la naturaleza de riesgo de la inversión, estos deben ser descontados a la tasa libre de riesgo en el modelo de valoración. 


\section{Aplicación.}

Con el propósito de implementar la metodología presentada en este documento se propone un proyecto hipotético sencillo de concesión vial (una carretera de peaje) en Colombia bajo un esquema BOMT (build-operate-maintenance-transfer, por su sigla en inglés) que se detalla a continuación. A través del esquema BOMT, el gobierno otorga el permiso a los inversionistas privados para financiar, construir, operar, mantener la infraestructura durante un periodo de tiempo determinado y luego, al final del periodo de concesión, estos transfieren la infraestructura sin contraprestación alguna.

\subsection{Acerca del proyecto de concesión, supuestos y resultados financieros.}

El proyecto de concesión involucra la construcción, operación, mantenimiento y transferencia de una carretera de doble calzada con longitud de $80 \mathrm{~km}$ al norte del país por un periodo de tiempo total de 15 años. Los primeros 2 años representan la fase de diseño y construcción y los próximos 13 años la fase de operación y mantenimiento. Al finalizar el periodo, la infraestructura es devuelta a la autoridad pública sin contraprestación alguna. Todas las cifras están expresadas en pesos colombianos (COP) al año 2018.

La construcción de la carretera comprende una inversión total de \$287,825 mil millones y será ejecutada a través de un contrato llave en mano. Esta inversión requerirá unos desembolsos anuales de $40 \%$ (año 1) y $60 \%$ (año 2). La inversión será financiada mediante un aporte de capital por parte de los inversionistas que comprende el $40 \%$ del monto total y un crédito sindicado con bancos locales por valor de \$ 196,145 mil millones a un plazo de 10 años. El valor total del crédito incorpora la actualización de los desembolsos por IPC durante los años de la construcción más los intereses de la fase de construcción. La tasa de interés del crédito tiene como base el índice de precios al consumidor (IPC) más un margen de 5\%, determinando así una tasa estimada a la fecha del $9 \%$. Además, la amortización del crédito se realiza bajo esquema de cuota fija que se empezará a pagar desde el primer año de operación. Las condiciones del crédito exigen una ratio mínima de cobertura de la deuda (DSCR) equivalente a 1.3 .

A partir del tercer año (primer año de operación del proyecto), la concesión tendrá a su cargo el funcionamiento de las casetas de cobro de peajes. La tarifa de peaje (promedio ponderado por categoría vehicular) autorizada a la fecha por la autoridad pública, la agencia nacional de infraestructura (ANI) corresponde a $\$ 17,691$, mientras el tráfico vehicular promedio (diario) estimado actualmente es de 4.080 vehículos en todas sus categorías (promedio ponderado). La Tabla 1 resume esta información y los demás supuestos del modelo financiero del proyecto.

Tabla 1. Supuestos del modelo financiero.

\begin{tabular}{lc}
\hline Duración & 15 años \\
Moneda & COP \\
Inflación (IPC): & $4 \%$ \\
Inversión total $^{\mathrm{i}}$,ii & $\$ 272.825$ \\
Costos de operación $^{\text {iii }}$ & $\$ 4.407$ \\
Gastos de administración $^{\text {iv }}$ & $10 \%$ \\
Tarifa de peaje $_{\text {Trafico promedio diario (TPD) }}$ & $\$ 17.691$ \\
Crecimiento anual del tráfico & 4.080 \\
Impuesto a la renta & $6 \%$ \\
Financiación $^{\text {Equity }}{ }^{\mathrm{v}}$ & $33 \%$ \\
Deuda $^{\mathrm{v}}$ & \\
\hline
\end{tabular}


i. La inversión total representa los recursos que necesita la infraestructura e incluye costos pre-operativos, estudios y diseños, compra de predios, capital de trabajo, costos financieros, interventoría, entre otros. ii-iii. Cifras en miles de millones de pesos.

iv. Se estiman sobre los ingresos brutos de recaudo de peaje.

v. Representa el aporte de los inversionistas privados e incorpora una tasa de rentabilidad mínima requerida del $12 \%$ (MARR).

Fuente: Elaboración propia.

Con estos supuestos, se procede a construir el modelo financiero del proyecto. La Tabla 2 resume los principales resultados del modelo financiero del proyecto, así como las estimaciones e indicadores de bondad (evaluación financiera), al igual que los ratios de cobertura (capacidad de pago) del proyecto.

Tabla 2. Estimaciones del proyecto.

\begin{tabular}{ccccc}
\hline Año & $\begin{array}{c}\text { Tráfico vehicular } \\
\text { esperado }\end{array}$ & $\boldsymbol{C F A D S}^{\mathbf{i}}$ & $\begin{array}{c}\text { Servicio de } \\
\text { deuda }\end{array}$ & DSCR \\
\hline 1 & - & - & - & - \\
2 & - & - & - & - \\
3 & $1,674,686$ & 40,362 & 30,564 & 1.32 \\
4 & $1,775,167$ & 44,226 & 30,564 & 1.45 \\
5 & $1,881,677$ & 48,501 & 30,564 & 1.59 \\
6 & $1,994,578$ & 53,230 & 30,564 & 1.74 \\
7 & $2,114,253$ & 58,460 & 30,564 & 1.91 \\
8 & $2,241,108$ & 64,244 & 30,564 & 2.10 \\
9 & $2,375,574$ & 70,638 & 30,564 & 2.31 \\
10 & $2,518,108$ & 77,706 & 30,564 & 2.54 \\
11 & $2,669,194$ & 85,518 & 30,564 & 2.80 \\
12 & $2,829,346$ & 94,150 & 30,564 & 3.08 \\
13 & $2,999,107$ & 103,688 & 30,564 & - \\
14 & $3,179,053$ & 114,225 & 30,564 & - \\
15 & $3,369,796$ & 125,865 & 30,564 & - \\
\hline i. Representa el flujo de efectivo disponible para cubrir el pago del servicio de \\
la deuda. \\
\hline
\end{tabular}

Fuente: Elaboración propia.

A partir de esta información, se estima el $V P N$ del proyecto utilizando una tasa de descuento ajustada por riesgo, es nuestro caso el $W A C C: 8,42 \%$, que refleja su estructura financiera. El WACC representa el costo promedio ponderado de capital del proyecto, a partir de la ponderación de los recursos de los inversionistas y la deuda contraída. Por simplicidad se asume una tasa de descuento constante para todo el periodo de evaluación. Como resultado se obtiene un VPN de \$19.297 y una TIR de $8,6 \%$, lo que indica que el proyecto es viable financieramente y, por tanto, los inversionistas, así como los prestamistas, estarán dispuestos a participar en su financiación. De igual forma, el proyecto muestra una capacidad de pago del servicio de deuda al encontrar un DSCR mínimo de 1,32 para todo el periodo, mientras su promedio asciende a 2,08. Aunque el modelo financiero del proyecto en su presentación determinista muestra resultados favorables, es necesario realizar un analisis estocástico de esté enfocado en la estimación de las probabilidades de incumplimiento. 


\subsection{Estimación de la probabilidad de incumplimiento.}

El primer paso para la estimación de la probabilidad de incumplimiento corresponde a la estimación de la volatilidad del $\left(\sigma_{D S C R}\right)$. Dada las dificultades que se presentan en su estimación se recomienda adoptar un enfoque similar al aplicado en la teoría de opciones reales (para más detalles ver Copeland \& Antikarov, 2001; Brandão, Dyer \& Hahn, 2012). Para ello, se asume que $\sigma_{D S C R}=\sigma_{C F A D S}$ según lo indicado en la sección 3. De esta $\sigma_{D S C R}$ reflejará la volatilidad total del proyecto. Bajo este enfoque se genera el mayor número posible de trayectorias de los flujos de efectivo del proyecto.

Al modelar los flujos de efectivo es posible reflejar si se puede generar un evento de incumplimiento, al incorporar las distintas fuentes de riesgo del proyecto, asociados a incertidumbres tanto operativas como de mercado, a partir de diversos supuestos probabilísticos que reflejen su propia naturaleza, así como sus correlaciones. Una ventaja de este enfoque, como indica Gatti (2008), corresponde a la representación de todas las fuentes de incertidumbre del proyecto. Por tanto, la volatilidad permite reflejar aquellos escenarios críticos. Esta misma se aplica en la teoría de opciones reales. Para ello, se debe adoptar un modelo adecuado de evaluación de riesgos, por ejemplo, se puede adoptar una estructura de desglose de riesgos. Al aplicar, por ejemplo, el modelo de Copeland y Antikarov (2001) se obtiene una volatilidad del 15\%.

Una vez se tiene la volatilidad $\left(\sigma_{D S C R}\right)$ se procede a estimar la prima por riesgo $\left(\lambda=\frac{\mu-r}{\sigma} \sqrt{T}\right)$, que al asumir una tasa libre de riesgo de $6 \%$ es equivalente a 0.2274 . El siguiente paso corresponde a la estimación de la distancia al incumplimiento $(D D)$ usando las ecuaciones (15) y (17) para cada año del periodo de amortización de la deuda. De igual forma, se define el punto de incumplimiento en sus dos versiones (estricto y técnico) según la ecuación (14), correspondientes a $D S C R_{t}<1$ y $D S C R_{t}<$ 1.3 (a partir de los supuestos del crédito sindicado acordado para el proyecto), respectivamente. La Tabla 3 resume los resultados obtenidos.

Tabla 3. Estimación de la probabilidad de incumplimiento.

\begin{tabular}{cccccccccccc}
\hline & & Año 1 & Año 2 & Año 3 & Año 4 & Año 5 & Año 6 & Año 7 & Año 8 & Año 9 & Año 10 \\
\hline \multirow{2}{*}{$D S C R$} & & 1.32 & 1.45 & 1.59 & 1.74 & 1.91 & 2.10 & 2.31 & 2.54 & 2.80 & 3.08 \\
\hline$D S C R<1,0$ & $D D$ & 1.62 & 2.06 & 2.47 & 2.84 & 3.18 & 3.50 & 3.78 & 4.04 & 4.28 & 4.50 \\
& $Q(t, T)$ & $8.21 \%$ & $3.35 \%$ & $1.26 \%$ & $0.45 \%$ & $0.16 \%$ & $0.05 \%$ & $0.02 \%$ & $0.01 \%$ & $0.00 \%$ & $0.00 \%$ \\
\hline \multirow{2}{*}{$D S C R<1,3$} & $D D$ & 0.61 & 1.14 & 1.63 & 2.07 & 2.48 & 2.86 & 3.21 & 3.52 & 3.81 & 4.07 \\
& $Q(t, T)$ & $35.14 \%$ & $18.12 \%$ & $8.11 \%$ & $3.25 \%$ & $1.20 \%$ & $0.42 \%$ & $0.15 \%$ & $0.05 \%$ & $0.02 \%$ & $0.01 \%$ \\
\hline
\end{tabular}

Fuente: Elaboración propia.

La Tabla 3 presenta dos resultados interesantes. En primer lugar, la diferencia entre utilizar un punto de incumplimiento estricto $\left(D S C R_{t}<1\right)$ y un incumplimiento técnico $\left(D S C R_{t}<1.3\right)$ dada la presencia de cláusulas restrictivas y covenants de la deuda. Estas diferencias se identifican mejor en la Figura 2. 
Figura 2. Estimación de la probabilidad incumplimiento.

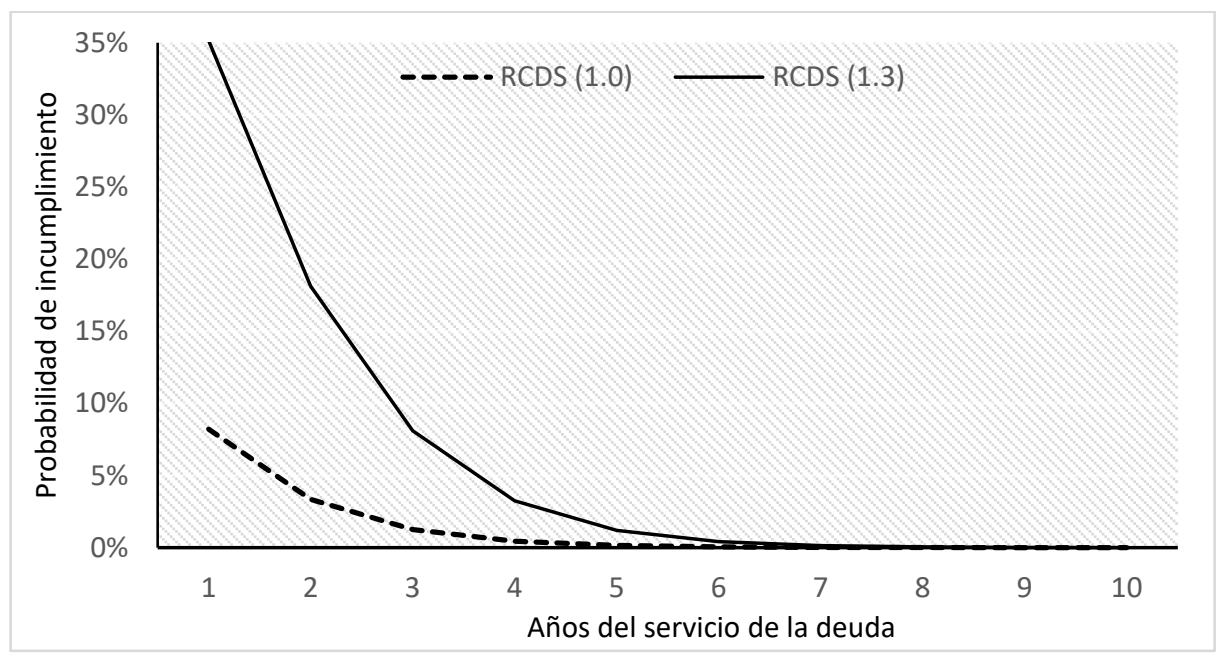

Fuente: Elaboración propia.

En su primer año, el proyecto alcanza el $D S C R$ más bajo de todo el periodo (1.32), por lo tanto, la probabilidad de incumplimiento en este año va a ser la más alta, mientras que a medida que mejora su capacidad de pago, es decir, aumenta la relación entre CFADS y el servicio de deuda para cada año $(t)$, la probabilidad va descendiendo gradualmente. Sin embargo, las diferencias que presenta el punto de incumplimiento (estricto: 1.0; técnico: 1.3) determinan diferentes niveles de probabilidad, con una diferencia de casi 27 puntos porcentuales. Esta diferencia se hace mínima al final del periodo de amortización cuando el $D S C R$ es superior a 2.5. El resultado también puede analizarse desde la $D D$, valores de este cercanos a cero reflejan una mayor probabilidad de incumplimiento.

De igual forma, la estimación de la $E L$ toma una dinámica similar a lo largo del periodo de amortización de la deuda, dada su relación directa con la probabilidad de incumplimiento. El hecho de que esta probabilidad descienda a niveles casi nulos al final del periodo indica que la $E L$ cae considerablemente. En otras palabras, sin tener en cuenta los otros dos componentes, el comportamiento asintótico que toma la probabilidad se refleja directamente en la $E L$. Sin embargo, este análisis está incompleto, dado que tanto la $L G D$ como la $E A D$, aunque están relacionados con la probabilidad, también tienen dinámicas propias como se analiza en la sección 5.

Finalmente, con el propósito de mostrar un análisis de sensibilidad de la probabilidad incumplimiento frente al parámetro de volatilidad, se realiza la estimación para todo el periodo de amortización del crédito, tomando diferentes volatilidades $(10 \%, 15 \%, 20 \% 25 \%)$, las cuales son asignadas hipotéticamente (solo con propósitos comparativos). Para ello, se parte solo de la definición del incumplimiento estricto $\left(D S C R_{t}<1\right)$. La Figura 3 muestra las estimaciones correspondientes para cada caso.

Lo anterior confirma la relación directa entre volatilidad y la probabilidad incumplimiento. Relación que es notable para los DSCR más bajos. A partir de estos resultados, se encuentra que la propuesta de Blanc-Brude y Hasan (2016) en la que proponen estimar la probabilidad de incumplimiento a través de un modelo estructural como una extensión de modelo KMV de Moody's representa una propuesta práctica y sencilla de aplicar con grandes aplicaciones en todos los sectores de infraestructura. 
Figura 3. Estimación de la probabilidad de incumplimiento.

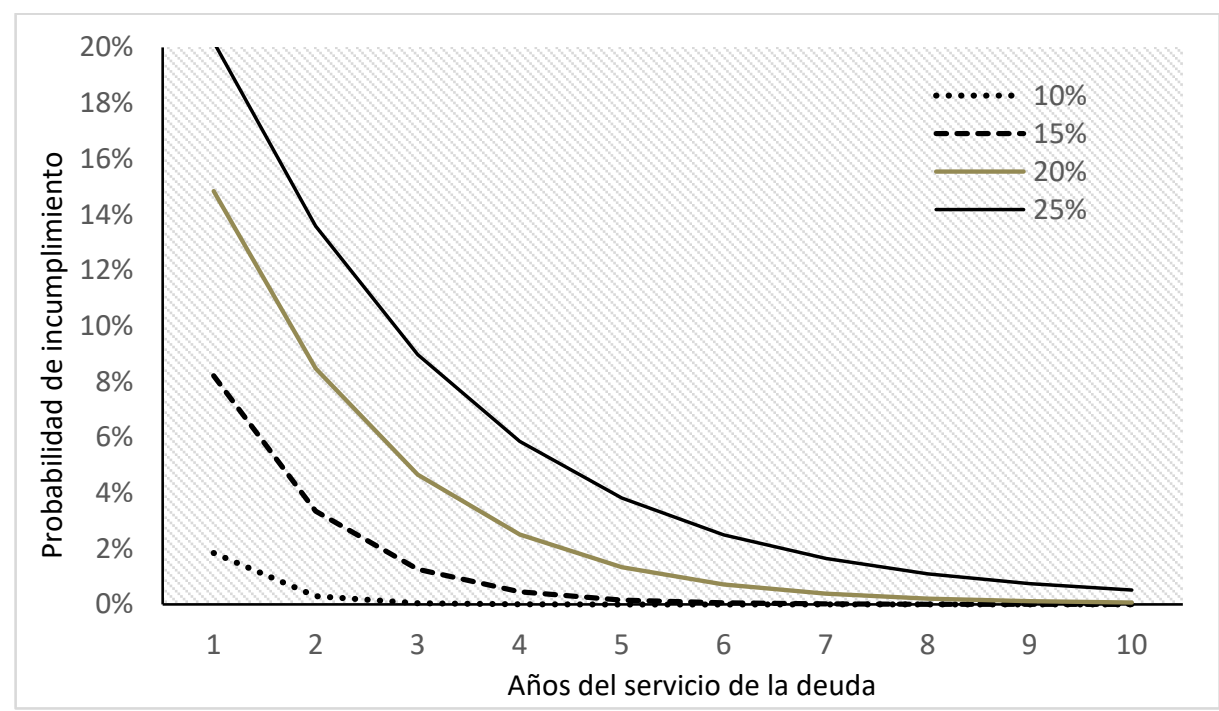

Fuente: Elaboración propia.

\section{Discusión final y conclusiones.}

Los modelos estructurales de riesgo de crédito para deuda corporativa asumen que el valor de la empresa es observable y que el incumplimiento ocurre cuando el valor de los activos cae por debajo del valor de la deuda. Éste es el principal insumo en el modelo de Merton (1974). Este desarrollo ha sido adaptado al tratamiento de riesgo de crédito en el campo de la financiación proyectos, especialmente inversiones en infraestructura, con resultados sorprendentes. Sin embargo, su aplicación merece un tratamiento cuidadoso, dado que el riesgo debe estimarse periódicamente durante toda la vida de la deuda, con el propósito de evaluar la dinámica de pago (capacidad de pago del servicio de la deuda) del proyecto a través del flujo de efectivo disponible.

Además, dada la naturaleza compleja de un evento de incumplimiento en campo de los proyectos, el tratamiento del riesgo de crédito está fundamentado en los umbrales definidos por la ratio de cobertura del servicio de deuda $(D S C R)$. Sin embargo, cuando se incumple este umbral (el nivel mínimo preestablecido), se debe tener presente que el evento no implica necesariamente un incumplimiento en sentido 'estricto', dada la presencia de covenants que llevan a la definición del incumplimiento 'técnico'. Por tal motivo, la definición de un umbral superior $(1 . x)$ puede utilizarse como un desencadenante para que, tanto prestamistas como patrocinadores, tomen las medidas necesarias para evitar el deterioro de las condiciones crediticias.

El análisis anterior debe extenderse para incorporar el efecto de una reestructuración del contrato de deuda en caso de un evento de incumplimiento. Dado que los prestamistas pueden aceptar una reestructuración en lugar de permitir que el proyecto incumpla el pago de la deuda, dadas las consecuencias adversas que esto produce. Estos efectos adversos incluyen la pérdida de calidad crediticia, el aumento de los costos de financiación y, por ende, una caída en el valor del proyecto. Según Gatti (2008), el contrato de deuda puede incorporar cláusulas que les permite a los prestamistas tomar medidas de control para evitar que la capacidad pago del proyecto se vea afectada. Por tanto, en un evento de incumplimiento, los prestamistas estarán dispuestos a tomar medidas correctivas para mantener el proyecto en normal funcionamiento, medida que incluso, puede generar una pérdida para ellos, dado que la re-estructuración puede extender los plazos de amortización, reducir el pago de intereses y honorarios o, por el contrario, acelerar su tasa de recuperación. Por tal razón, los componentes del riesgo de crédito ( $L G D$ y $E A D)$ pueden cambiar en un escenario de este tipo, por lo 
que los modelos de estimación deben incorporar estos ajustes. Es común que estas medidas correctivas les permita renegociar las condiciones actuales de la deuda, cambiando los plazos de amortización, el pago de intereses, la incorporación de créditos subordinada. Incluso, los prestamistas pueden llegar a sustituir a los contratistas, operadores y proveedores, cuando las condiciones lo merecen.

La incorporación de eventos de re-estructuración requiere determinar, en caso de un incumplimiento, cómo los factores desencadenantes afectan la estimación de los componentes de $L G D$ y $E A D$. Con respecto a la $L G D$, se sabe que la tasa de recuperación depende claramente de los $C F A D S$ futuros y, por tanto, del valor del proyecto en caso de incumplimiento. Aunque su aplicación puede ser compleja, estos se pueden incorporar a partir de la descomposición de Black-Cox en el modelo estructural propuesto por Blanc-Brude y Hasan (2016). La descomposición de Black-Cox, propuesta por Black y Cox (1976), comprende un método utilizado para valorar deuda corporativa en escenarios de reestructuración o refinanciación cuando el valor de los activos de la empresa alcanza un límite (inferior o superior). De esta forma, el modelo de estimación debe requerir de un ajuste adicional, dado que solo contempla la dinámica que proporciona el flujo de efectivo.

Este ajuste se presenta a partir de una descomposición que incorpora cuatro funciones de pago dadas a continuación:

1. $P\left(T_{D}, C F A D S_{T_{D}}\right)$ : pago final al vencimiento de la deuda, donde $T_{D}$ representa el vencimiento de la deuda.

2. $\underline{P}\left(\tau, \underline{C F A D S_{\tau}}\right)$ : valor de los activos si el CFADS alcanza el límite inferior, es decir, un incumplimiento, en el tiempo $\tau$, llevando a un estado de reestructuración.

3. $\bar{P}\left(\tau, \overline{C F A D S_{\tau}}\right)$ : valor de los activos si el CFADS alcanza el límite superior en el tiempo $\tau$ y corresponde a un estado de refinanciación, debido a que permite acelerar el pago de la deuda, junto con una reducción en sus costos.

4. $p^{\prime}\left(t, C F A D S_{t}\right)$ : pago de la deuda realizado antes del vencimiento o la reestructuración.

Así, el valor total de los activos corresponde al valor presente (esperado) de la suma de las cuatro funciones de pago, bajo la medida de probabilidad riesgo-neutral. Si se define $h\left(V_{t}, t\right)$ como el valor de los activos en un momento $t$ y $K(\cdot)$ denota el intervalo $\left(\underline{C F A D S_{\tau}}(\cdot), \overline{C F A D S_{\tau}}(\cdot)\right)$, y $V_{t}$ es el valor de todos los flujos de efectivo futuros, entonces para cada función de pagos se tiene:

$$
\begin{gathered}
h_{1}\left(V_{t}, t\right)=E\left[e^{-r_{T_{D, t}}\left(T_{D}-t\right)} P\left(T_{D}, C F A D S_{T_{D}}\right)\right] \\
=e^{-r_{T_{D, t}\left(T_{D}-t\right)} \int_{k(T)}\left(T_{D}, C F A D S_{T_{D}}\right) d F^{*}} \\
h_{2}\left(V_{t}, t\right)=\int_{t}^{T} e^{-r_{\underline{C A D S}, t}\left(T_{\underline{C F A D S}}-t\right)} \times \underline{P}\left(C F A D S_{T_{\underline{C F A D S}}}, T_{\underline{C F A D S}}\right) d F_{T_{\underline{C F A D S}}^{*}} \\
h_{3}\left(V_{t}, t\right)=\int_{t}^{T} e^{-r_{\overline{C F A D S}, t}\left(T_{\overline{C F A D S}}-t\right)} \times \bar{P}\left(C F A D S_{T_{\overline{C F A D S}}}, T_{\overline{C F A D S}}\right) d F_{T_{\overline{C F A D S}}^{*}} \\
h_{4}\left(V_{t}, t\right)=\int_{t}^{T_{D}} e^{-r_{S, t}(s-t)} \times\left[\int_{k(T)} p^{\prime}\left(C F A D S_{S}, s\right) d F^{*}\left(C F A D S_{S}, s\right)\right] d s
\end{gathered}
$$

donde $d F^{*}$ es la probabilidad de que $C F A D S$ descienda del umbral durante $T_{D}, F_{T_{C F A D S}}^{*}$ define la función de densidad cuando el $C F A D S$ alcanza el límite de incumplimiento, mientras $F_{T_{\overline{C F A D S}}^{*}}^{*}$ define la función de densidad cuando alcanza el límite superior (re-financiación). 
De esta forma, el valor total de los activos $\left(V^{S}\left(V_{t}, t\right)\right)$ será determinado por:

$$
V^{s}\left(V_{t}, t\right)=\sum_{i=1}^{i=4} h_{i}\left(V_{t}, t\right)
$$

donde $h_{i}\left(V_{t}, t\right)$ es el valor determinado por la i-ésima función de pagos.

Dada la complejidad en la implementación en la descomposición de Black-Cox, Gatti (2008) propone un enfoque alternativo a partir de la técnica de simulación de Monte Carlo. Sin embargo, esta requiere una aplicación de varios niveles o etapas de simulación. Una vez se obtienen los resultados del modelo de Monte Carlo (en su primera etapa), para estimar la probabilidad de incumplimiento, se debe ejecutar un conjunto de simulaciones para cada escenario por incumplimiento, con el objetivo de obtener la distribución de los valores de $L G D$. Gatti (2008) también señala que se puede emplear un ejercicio más simple de estimación para evitar la complejidad computacional. En este caso, se puede asumir un valor fijo de la $L G D$ mientras se modela el valor del proyecto en caso de incumplimiento (etapas 1 y 2). Sin embargo, esta alternativa genera un problema de subestimación en el riesgo, dado que omite la correlación positiva que existe entre la probabilidad de incumplimiento y la $L G D$ : una caída de los flujos de efectivo aumenta directamente la probabilidad de incumplimiento, al tiempo que reduce la tasa de recuperación para los prestamistas. Además, como señala Gatti (2008), este ejercicio puede proveer información adicional relevante para el análisis de riesgos del proyecto, como lo es el VaR definido a un nivel de confianza (por ejemplo, 99.9\%) y un horizonte de tiempo determinado, o para cada año de vida de la deuda.

Este tipo de aplicaciones usando la técnica de simulación de Monte Carlo es utilizada por Gatti et al. (2007) para la estimación del valor en riesgo ( $\mathrm{VaR}$, por su sigla en inglés) del proyecto. Sin embargo, el lector debe tener presente que la modelación de factores de riesgo representa un tema crítico, donde los juicios y criterios propios tienen un gran efecto en la evaluación de riesgo y, por ende, en la estimación de la probabilidad de incumplimiento.

\section{Referencias}

Aragones, J., Blanco, C., \& Iniesta, F. (2009). Modelizacion del riesgo de credito en proyectos de infraestructuras. Innovar, 19(35), 65-80.

Basel Committee on Banking Supervision (2004). Basel II: International Convergence of Capital Measurement and Capital Standards: a Revised Framework. Bank for International Settlements, Basel.

Black, F., \& Cox, J. (1976). Valuing corporate securities: Some effects of bond indenture provisions. The Journal of Finance, 31(2), 351-367.

Black, F., \& Scholes, M. (1973). The Pricing of Options and Corporate Liabilities. The Journal of Political Economy, 81(3), 637-654.

Blanc-Brude, F., \& Hasan, M. (2016). A Structural Model of Credit Risk for Illiquid Debt. The Journal of Fixed Income, 26(1), 6-19.

Blanc-Brude, F., Hasan, M., \& Ismail, R (2014). Unlisted Infrastructure Debt Valuation \& Performance Measurement. EDHEC-Risk Institute Publications.

Blanc-Brude, F., Hasan, M., \& Whittaker, T. (2016). Cash Flow Dynamics of Private Infrastructure Project Debt: Empirical evidence and dynamic modelling. EDHEC-Risk Institute Publications. 
Blanc-Brude, F., Hasan, M., \& Whittaker, T. (2018). Calibrating credit risk dynamics in private infrastructure debt. The Journal of Fixed Income, 27(4), 54-71.

Blanc-Brude, F., \& Strange, R. (2007). How Banks Price Loans to Public-Private Partnerships: Evidence from the European Markets. Journal of Applied Corporate Finance, 19(1), 94-106.

Borgonovo, E., \& Gatti, S. (2013). Risk analysis with contractual default. Does covenant breach matter? European Journal of Operational Research, 230(2), 431-443.

Boussabaine, A. (2013). Risk pricing strategies for public-private partnership projects. New York: John Wiley \& Sons.

Brandão, L., Dyer, J., \& Hahn, W. (2012). Volatility estimation for stochastic project value models. European Journal of Operational Research, 220(3), 642-648.

Buscaino, V., Caselli, S., Corielli, F., \& Gatti, S. (2012). Project finance collateralised debt obligations: An empirical analysis of spread determinants. European Financial Management, 18(5), 950-969.

Cartea, Á., \& Figueroa, M. (2005). Pricing in Electricity Markets: A Mean Reverting Jump Diffusion Model with Seasonality. Applied Mathematical Finance, 12(4), 313-335.

Copeland, T., \& Antikarov, V. (2001). Real Options: A practitioner's guide. New York: Texere Publishing Limited.

Della, R., \& Yermo, J. (2013). Institutional investors and infrastructure financing. OECD Working Papers on Finance, Insurance and Private Pensions, No. 36, OECD Publishing.

Dong, F., Chiara, N., Kokkaew, N., \& Xu, A. (2012). Copula-Based Portfolio Credit Risk Assessment in Infrastructure Project Financing. Journal of Private Equity, 15(2), 31-40.

Freydefont, M. (2001). An Approach to Credit Risk Valuation for Structured and Project Finance Transactions. Journal Of Project Finance, 6(4), 53-67.

Gatti, S. (2008). Project finance in theory and practice: designing, structuring, and financing private and public projects. Oxford: Academic Press.

Gatti, S., Rigamonti, A., Saita, F., \& Senati, M. (2007). Measuring Value-at-Risk in project finance transactions. European Financial Management, 13(1), 135-158.

Grimsey, D., \& Lewis, M. (2002). Evaluating the risks of public private partnerships for infrastructure projects. International Journal of Project Management, 20(2), 107-118.

Ingersoll, J. (1977). A contingent-claims valuation of convertible securities. Journal of Financial Economics, 4(3), 289-321.

Karminsky, A., \& Morgunov, A. (2016). The assessment of the credit risk of investment projects. XVI прельская международная научная конференция по проблемам развития экономики и общества. Moscú, National Research University Higher School of Economics, 721-731.

Kealhofer, S. (1993). Portfolio management of default risk. KMV Corporation.

Klompjan, R., \& Wouters, M. (2002). Default Risk in Project Finance. The Journal of Structured Finance, 8(3), 10-21. 
Kong, D., Tiong, R., Cheah, C., Permana, A., \& Ehrlich, M. (2008). Assessment of Credit Risk in Project Finance. Journal of Construction Engineering and Management, 134(11), 876-884.

McQuown, J. (1993). Market vs. accounting based measures of default risk. KMV Corporation.

Merton, R. (1973). Theory of Rational Option Pricing. Bell Journal of Economics and Management Science, 4(1), 141-183.

Merton, R. (1974). On the Pricing of Corporate Debt: The Risk Structure of Interest Rates. The Journal of Finance, 29(2), 449-470.

Vasicek, O. (1984). The philosophy of credit valuation: the credit valuation model. KMV Corporation.

Yescombe, E. (2002). Principles of Project Finance. San Diego: Academic Press. 